\title{
Understanding Student Use of Resources in "Rich-Media" Courses
}

\author{
T. L. Rodgers*, S. Mabley, and A. A. Garforth \\ The University of Manchester
}

\begin{abstract}
There is a current trend in course development to increase the number of "Rich-Media" items available to students; these include items like key-concept videos, interactive activities and quizzes, and even captures of the full lectures. It is therefore important to understand which of these resources students use and gain value from so that we can target the best resources for student learning.

This paper looks at several courses taught in the School of Chemical Engineering and Analytical Science at The University of Manchester to several year groups; including lecture based courses, distance learning courses, and non-lecture based activities; that contain "Rich-Media" resources. The use of these items by students is examined; including number of uses, time of use, and local retention. The student opinion on the items and how they felt they affected their learning is also analysed. This allows results to be presented on the most useful types of resource for students providing information for future development.
\end{abstract}

Keywords; rich-media, chemical engineering, student engagement.

*Correspondence to: The Mill. School of Chemical Engineering and Analytical Science, The University of Manchester, Manchester, M13 9PL.E-mail: tom.rodgers@manchester.ac.uk

\section{INTRODUCTION}

In recent years the number of "rich-media" resources in engineering education has increased. This is likely due to the improvement of technical possibilities (for example, recording software and automatic lecture capture systems), improvement of distribution options (YouTube, tablets and, virtual learning environments), and demand from increasing student expectations. Therefore, engineering higher education has moved from the situation where using rich-media to support teaching was rare to one where it is becoming more common place, with an increasing number of institutions bringing in recorded lecture capture.

Current research emphasizes that video lectures can provide important benefits to students (Giannakos et al., 2015) as video lectures offer students the ability to review the lecture at their own pace. They also allow students who miss the live lecture to have the opportunity to catch up on the material. This is especially useful for content-heavy disciplines such as chemical engineering, where significant amounts of detailed information may be presented during each live lecture. Fernandez et al. (2009) also comment that providing students with access to 
recorded lectures allows universities to engage with students who study by different learning methods and often increases students' motivation and sense of interaction with the lecturers.

Despite this, research into how students use rich-media resources, and which type of these resources are most used by the students is still largely limited, many studies focus on student opinion of the material and attendance. Case studies on the use of rich-media in higher education have been presented across a range of subjects including business, psychology, and engineering. The rich-media material considered in these studies is also in a variety of formats including audio-only podcasts, enhanced podcasts, video segments, or full lecture capture. It is also important when examining the use of rich-media resources to consider also the pedagogy as well as the preference of the students and the purpose of the resource; this is mentioned in Van Zanten et al. (2012) with their 3Ps and has been recently extended by Saunders and Hutt (2015) to include a fourth $\mathrm{P}$ of the performance of the students.

The discussion in this paper focuses on the approach where rich-media resources are used to accompany traditional teaching methods, i.e. lectures and tutorials, rather than replace these traditional methods, e.g. MOOCs. This discussion has arisen to address a small number of students not engaging with rich-media material either because they are unwilling or unable; similar to Kazlauskas and Robinson (2012) who saw up to a fifth of students in a cohort not engaging. In this approach rich-media materials supplemented the existing course; allowing students either the opportunity to re-engage with already presented lecture content and/or to access supplementary course content (Saunders and Hutt, 2015). This of course means that pedagogical decisions are essentially limited to the material content and format.

The aims of rich-media materials vary considerably; including assignment preparation (Belton, 2016; Copley, 2007; Parson et al., 2009; Sutton-Brady et al., 2009), revision materials (Copley, 2007; Davis et al., 2009; Pearce and Scutter, 2010; Van Zanten et al., 2012), improving understanding (Bongey et al., 2006; Leadbeater et al., 2013; Parson et al., 2009; Pearce and Scutter, 2010; Van Zanten et al., 2012) and, lecture capture (Davis et al., 2009; Leadbeater et al., 2013; Parson et al., 2009; Pearce and Scutter, 2010); as did student use of the materials. To fulfil these aims rich-media materials have been created involved pod-casting (both audio and video), the use of narrated PowerPoint slides, short video segments, and lecture capture (either audio only or video plus audio).

There is still little documented evidence as to how effectively students are actually using these technologies, e.g. is student learning improved. Guertin et al. (2007) even commented that students who claim they want the material may not even use it; however, this may have been due to many students not knowing they could retrieve the recordings.

Recording lectures is thought of by many to enable students to skip lectures, though there seems little documented evidence either way. This is maybe due to the fact that although recorded lectures allow students to "attend" a lecture whenever they want, the material still takes the same time as the original lecture, and doesn't allow the students to interact with the lecturer. Holbrook and Dupont (2009) found no significant correlation between declining attendance and the ability to access recorded lectures. Pursel and Fang (2012) reviewed a large number of articles and found that there was no discernible link between provision of lecture captured materials and 
declining attendance from both self-reporting studies and from actual attendance data. Franklin et al. (2011) actually reported a small increase in attendance in those modules that provided captured lectures. They also commented that students will miss lectures with or without the provision of lecture capture and that their decision is based on previous experience of the lecturer's style or their own personal learning method.

It is clear that rich-media material has become part of the expectation in higher education teaching and that there are examples of material designed that students like. However, there are gaps in our understanding of how students use these materials and comparison of the different styles of material available. Therefore, this study uses a combination of data to address the following questions:

- How do students interact with rich-media resources?

- Which type of rich-media resources do students use the most?

- Do students like rich-media resources?

- Does rich-media use effect exam results?

- Do people who don't attend the lecture use the rich-media resources more?

- Can we create rich-media to replace some taught content?

The results presented here provide information on how students use rich-media resources and what type of rich-media resources should be developed further for students.

\section{METHOD}

This study examined student use of the rich-media material provided in two, simultaneously taught, third year undergraduate and MSc Chemical Engineering lecture based units and an undergraduate laboratory based unit delivered at The University of Manchester, UK. The first lecture based unit was "Advanced Engineering Separations" (AES) and the second unit was "Catalytic Reaction Engineering" (CRE) both delivered between September 2015 and January 2016 (Semester 1). Both modules were delivered to a cohort of 202 third year undergraduate students and $26 \mathrm{MSc}$ students, and consisted of weekly lectures. The majority of the students within the class were aged between 20 and 25. The laboratory based unit was the first year core chemical engineering labs (LABS) delivered between September 2015 and May 2016 (Semester 1 and 2). LABS was delivered to a cohort of 285 first year undergraduate students, with majority of the students aged between 18 and 21 .

The undergraduate students were familiar with the use of full recorded lectures as around $85 \%$ (higher in first and second year units) of the Chemical Engineering units delivered at The University of Manchester are automatically recorded. Approximately $42 \%$ of the undergraduate students and $96 \%$ of the MSc students were international students.

The rich-media material provided for these modules consisted of six different types:

1. Full lecture podcasts that were recorded automatically. The podcasts captured sound from the lecture theatre microphone and video from the lecture theatre projection system (power point presentations). These recordings were edited to remove pauses for breaks and tutorial sessions. Each was made available to students the day after the lecture was delivered via the university VLE (Blackboard). AES consisted of 10 of these at an average of 47 minutes in length. 
2. Tutorial solution videos. These were worked tutorial solutions with voice-over audio explaining each step, they consisted of a mixture of hand-written recordings and the use of power point with excel depending on how the question needing to be solved. They were made available within the unit VLE at the end of the week the material was taught. AES consisted of 7 of these at an average of 11.4 minutes in length.

3. Key-concept videos. These videos were short and each examined one concept associated within the module in a focussed manner, and were selected based on topics the students had struggled with in previous years. They were made available within the module VLE at the start of the week the material was taught. The videos were produced as power point presentations with animations. AES consisted of 6 and CRE consisted of 3 of these at an average of 5.5 minutes in length.

4. Revision lecture video. This video was the live recording of a revision session where a past exam paper was answered. This video was 102 minutes in length.

5. Chapter summary videos. These videos provided a summary of each chapter in the unit handbook as power point presentations. They were made available within the module VLE at the end of the week the material was taught. CRE consisted of 9 of these at an average of 19 minutes in length.

6. Laboratory study videos. These videos provided a summary of the context, theory, experimental method, and learning outcomes of a particular experiment. These videos were produced as animations with videos of laboratory equipment. LABS consisted of 5 of these at an average length of 5 minutes.

Data was also available from the AES course for September 2014 to January 2015 (AES2014) with only full lecture captures recorded. In this year there was 165 third year undergraduate chemical engineers with 115 being an in house class for the live lectures and 50 being distance learners with no option to attend the live lectures.

After the 2015-2016 courses the materials were made publically available and watched by people not enrolled on the courses, this means that viewing behaviour cannot be compared with the 2016-2017 classes. The one exception to this was the new lectures recorded for the AES course for September 2016 to January 2017 (AES2016); these were only made available to the class via YouTube. In this year the AES course was taken by 258 third year undergraduate chemical engineers (217 in house and 41 distance learners) and 59 MSc students.

To understand students' use of the videos, data from YouTube analytics was collected. This provided fine-grained data on the use of the videos including number of views, percentage watched, demographics, device used, and retention (the number of people watching at a given time divided by the number of people who start to watch the video, expressed as a percentage). The videos were unlisted so were only accessed through the links in Blackboard. Comments about the videos were also examined on student questionnaires about the courses, these had a return rate of approximately 50\%. For the lecture videos as well as being available on YouTube, they were also available via the university's own system which gave information on the number and times of views. For AES, in 2014 the full lecture capture recordings were made available through the Blackboard system as webpages, however due to this implementation only data on interactions with the videos was available, but this was available for individual students (unlike the YouTube analytics data). 


\section{RESULTS}

\subsection{Student Views}

The number of views of the videos were high with 6948 views ( $\sim 30$ per student) for the AES videos and 4989 views ( 22 per student) for the CRE videos. This means on average that each video for AES was watched around 278 times (approximately 1.2 times per student) while each video for CRE was watched on average 415 times (approximately 2 times per student). This larger number of views for the CRE videos could be due to there being 12 videos available compared to 24 for AES. Table 1 shows the breakdown of the number of views for each video type.

Figure 1 shows how these number of views break down against days from the start of the units. These usage curves are typical of all the resources made available to students - a steady usage during the module with some minor spikes at key times with a sharp spike immediately before the exam in each module. For example, Lecture A, Tutorial A, and Concept A are videos all related to the first coursework test (due $1^{\text {st }}$ November) and there is clearly higher use up to this time and then a marked drop in the number of views until late December when the videos were being used for exam revision. The same pattern is seen in the Lecture B, Tutorial B, and Concept $\mathrm{B}$ videos which are all related to the second coursework test $\left(6^{\text {th }}\right.$ December). It can also be seen that the tutorial videos are watched over a wider range of time whereas the lecture and concept videos are mainly watched during revision periods (test and exam preparation). The watching of the tutorial videos corresponds to when students were undertaking the relevant tutorial question, which depending on the student varied over several weeks. Although no survey of use was undertaken, when asked, students commented that they either used the tutorial videos to check their answer or watched the relevant answer part to a question they were unable to answer.

During the time the students revised for their exams the number of video views spiked, this period of time is approximately 4 weeks. There is a gap in the number of views for both the AES and CRE videos. The first gap is around the $20^{\text {th }}$ January as many of the students in the modules had another exam, while the gap in the CRE video views continues in the few days before the AES exam on the $22^{\text {nd }}$ January.

If students can be said to be picking their favourite video type or most useful video type by how many times it is viewed, then the video types can be ranked by number of views. This would mean that the "best resource" is the chapter summary type followed by the concept videos, and this agrees with opinions from the students discussed in Sadik (2015) who said that they preferred short screencasts to full lecture capture.

Comments from the students backed up the fact that all the video types were used. For the AES course, which has a selection of video types available, students commented, "Additional short videos worked well alongside the lecture material and clarified the main points outlined in each lecture.", “... also added video solutions to some questions, thank you very much, these are great help." 
Table 1. Summary of the average video length, views, and retention for the AES and CRE videos from YouTube, and the number of lecture views on the university (UoMan) system. AES2016 lecture number of views data is scaled by the number of students in 2015 to number of students in $\mathbf{2 0 1 6}$ to provide a fair comparision.

\begin{tabular}{|l|cccc|}
\multicolumn{1}{c|}{ Video Type } & $\begin{array}{c}\text { Number of } \\
\text { videos }\end{array}$ & $\begin{array}{c}\text { Average } \\
\text { length / min }\end{array}$ & $\begin{array}{c}\text { Average number } \\
\text { of views per video }\end{array}$ & $\begin{array}{c}\text { Average } \\
\text { retention / \% }\end{array}$ \\
\hline Revision (AES) & 1 & 102.4 & 201.0 & 19.5 \\
Concept (Both) & 9 & 5.5 & 276.6 & 64.5 \\
Lecture (AES) & 10 & 47.3 & 195.2 (YouTube) & 37.3 \\
Lecture (AES2016) & 9 & 52.6 & 188.7 (UoMan) & 38.8 \\
Tutorial (AES) & 7 & 11.4 & 243.1 & 50.2 \\
Chapter (CRE) & 9 & 19.0 & 411.8 & 55.2 \\
\hline
\end{tabular}




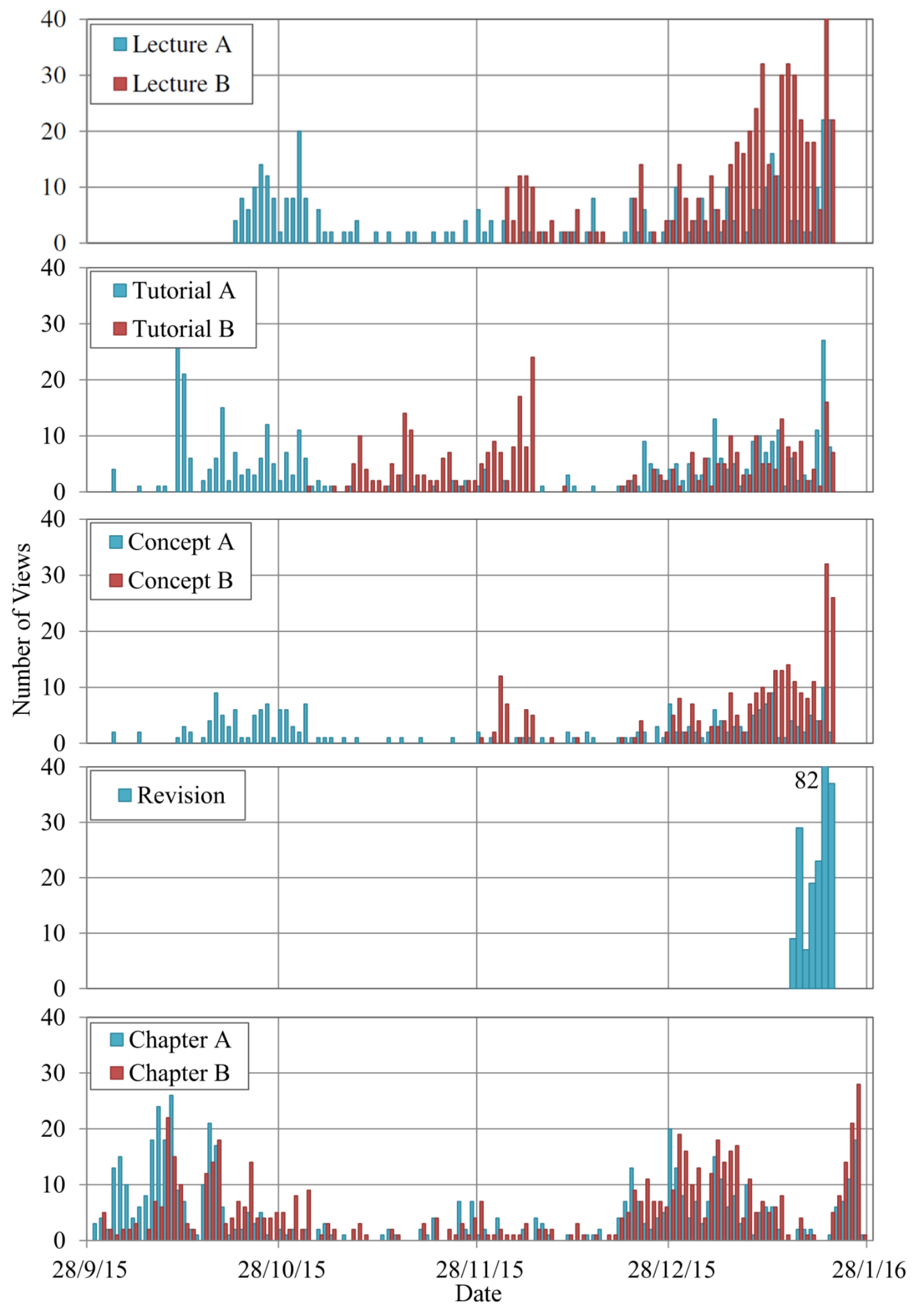

Figure 1. Number of views for selected average videos for the 5 video types. 


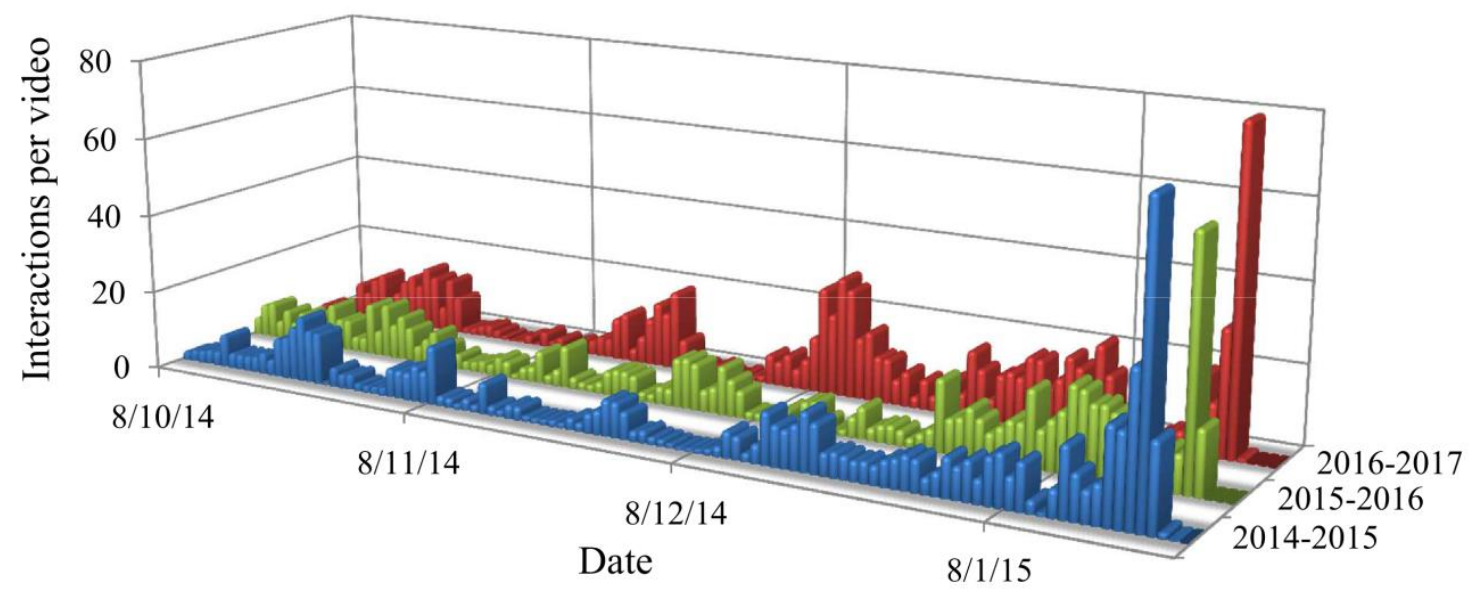

Figure 2. Number of daily interactions for the AES lecture videos, showing the similarity over the three years. The dates have been adjusted for 2015 onwards to line up the day of the final exam, which is within the same week in January. All data is scaled by student number to make it equivalent to 2014-2015.

Although the videos were designed for the specific courses it should be noted that students are still using the videos. For example the AES videos were viewed 286 times after the end of the course by the end of semester 2 with $47 \%$ of these views on two of the videos which are most related to the design project that the same students undertake in semester 2 .

As a comparison with the data collected for the lecture videos for other years, we can see these averaged per video in Figure 2 and shows very similar behaviour between the uses of the lecture videos in all years. It can also be noted that taking an average of 2.8 interactions per view scales the number of views (for AES2015, and AES2016) to number of interactions per video (for AES2014) well. This means that we can use the AES2014 per student data to help draw conclusions about the uses of rich-media.

\subsection{Video Retention}

The viewing figures do hide some details; for example, on average only $64 \%$ of a key concept video was watched (Table 1). The percentage for the lecture videos was lower with about $37 \%$ of each video being watched. Interestingly there is no real difference in percentage of video viewed if the retention data is split into the period during the units and the period for revision, meaning that there is very little difference in the way students were using the materials.

Data from YouTube analytics allowed viewing patterns of the videos to be studied; Figure 3 shows the number of views of each segment of the video as a percentage of initial viewers (local retention). An increase in the percentage with time indicates either that viewers skipped to this point in the video, or that they rewound to this section to watch again. The viewing patterns are different for the different types of video; however, there is commonality that there is a rapid loss of audience in the first few seconds. This is most likely due to people quickly moving to the 

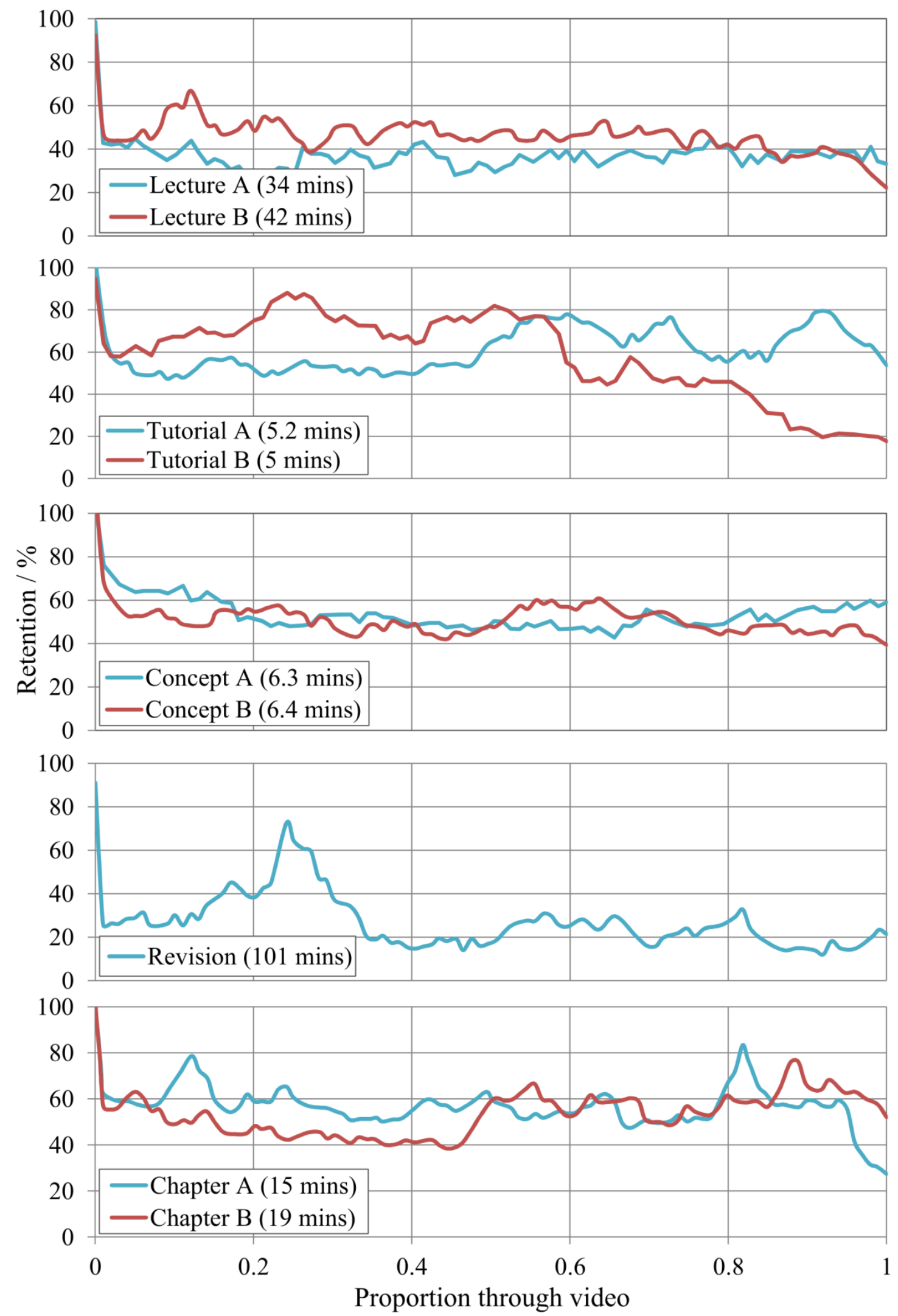

Figure 3. Retention for selected average videos for the 5 video types. 
part of the video they want to watch, or simple not watching the video. For the lecture and key concept videos the viewing then stays fairly constant for the rest of the video. Small fluctuations in the retention are most likely due to students rewinding or skipping small sections. The average retention for the key concept videos is higher than that of the lecture videos, and this is most likely due to two reasons. The first that the concept videos were much shorter than the lecture videos, thus quicker to watch; several students commented that they watched the lecture videos in 2 or 3 attempts. The second that the concept videos were picked for areas that the students struggled with in previous years and only provided the most important information. There is also no difference between the style of patterns of local retention for the AES lectures in 2015-2016 to those for the AES lectures in 2016-2017.

The chapter summary videos have a similar retention profile to the concept videos; however, there are sections of the video that have higher retention, for example Chapter B video (Figure 3) where the second half has around $20 \%$ higher number of views than the first half and can be explained by this part dealing with more difficult material. The tutorial solution videos and the revision session video (essentially a long tutorial video) have a different retention profile compared to the other video resources. There are more fluctuations in the watching of the videos, this is due to students skipping to or rewinding back to key areas and agrees with, the previously mentioned student comments that they either used the tutorial videos to check their answer or watched the relevant answer to question they were unable to answer. Therefore, one would expect the retention of the tutorial videos to be lower as some students are only looking at the answer sections and not the method on how to answer the question. This effect is also seen in the revision video where there is a large spike at the start of the most difficult question.

By noting the timing of any peaks or troughs in the retention curves, it is possible to link these to events in the videos allowing for analysis or identification of the causes of what makes viewers stop viewing or skip material. Unclear parts of a video were associated with a drop in viewers, whereas the start of tutorial questions, the final reveal of answers, and difficult sections were associated with an increase in viewers.

These results again suggest a preference for the short key concept videos and the chapter videos as the viewing figures were higher and the retention was higher, though the key concept videos have the highest retention. Although the retention was lower for the full lecture recordings it should be noted that the one item the students asked for were recordings of the full lectures. This was most likely as it allowed them to review any of the lecture material if they needed to as a back up to other methods, for example, to cross-reference their own notes taken at the time.

As the videos provided were of a different length and it is known that the retention of a video tends to decrease with length, Figure 4 details the length versus retention for each AES and CRE video. This should hopefully allow an analysis of if the key factor is just length, i.e. related to the student's comments of watching a lecture recording in multiple views.

Figure 4 shows that there is a trend between the video length and the retention to which a trend line can be fitted. The best fit was given by an exponential decay which provides an adequate fit to the data (the $\mathrm{R}^{2}$ value is larger than the value needed for $0.001 \%$ significance for 


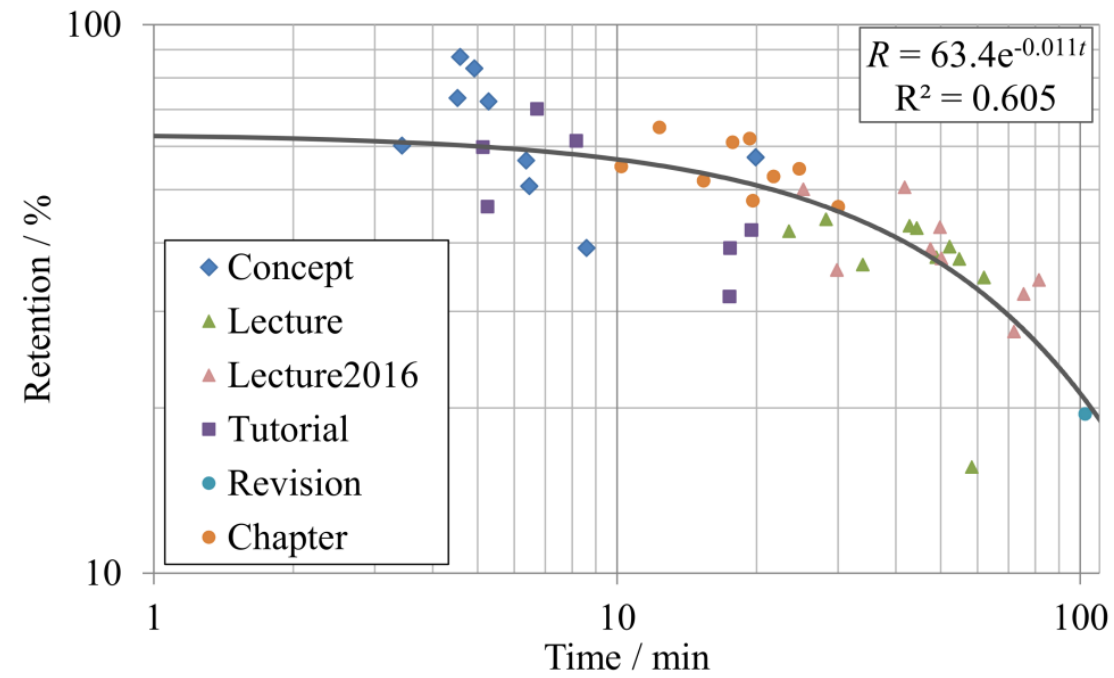

Figure 4. Average retention vs video time for all the 2015 AES and CRE videos, plus the AES2016 lecture videos.

this number of data points, $\sim 0.5)^{1}$. This trend line could then be used as some kind of average benchmark for the videos; those above the line are essentially retaining people better than those below. A key point to note is that the trend line does not pass through $100 \%$ at a theoretically 0 second video (it would be $63.4 \%$ ), this reflects the data seen in Figure 3 at short times, that there is a quick drop off of in viewing. This is most likely due to students either selecting an incorrect video, or YouTube's autoplay function that some students may have turned on, and then turning the video off quickly. Another key point about the trend line is that although it asymptotes to $0 \%$ retention, for all purposes any video longer than 500 minutes would have $0 \%$ retention.

From Figure 4 some key information about the video design can be inferred, the first is that long tutorial style videos are comparatively worse for retention. The most likely cause being too much information is trying to be delivered in these videos and they should potentially be spilt into a key concept and a shorter tutorial video, as students are not looking for this level of information in this location. The second is that, although important to the unit, the lecture that contains the introduction to the unit and information about how the unit will work is poorly watched (the lecture with $13 \%$ retention). The result being that important information within this lecture may be lost to the students and therefore the unit introduction should either be separated into a separate clearly labelled video or perhaps not even provided as a video. This same lecture from AES2016 had the introduction information removed from the recording; the retention for this version is in line with the other retention data.

\subsection{Distance Learning}

The AES course contains some students who are distance learners and as such don't attend the lectures in person potentially giving an insight into any difference in the use of the videos dependant on attendance at the lectures or not. It should be noted that the average attendance for the AES course across the whole semester for the people that were not distance learners, the in-

\footnotetext{
${ }^{1}$ It should be noted that removing the revision lecture point from the graph does not actually change the fitted trend line.
} 


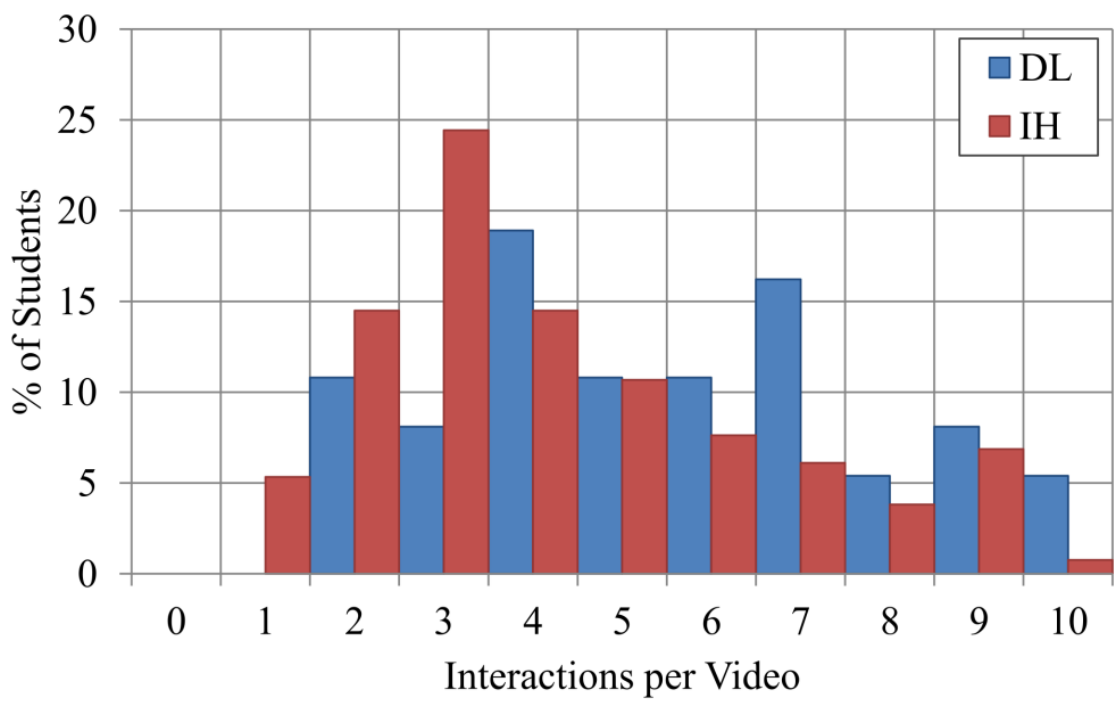

Figure 5. Interactions with the AES2014 lecture videos, distance learners (DL) vs in-house class (IH).

house class, was $83 \%$. The information could be examined for the AES2014 class as the number of interactions could be retrieved per student, Figure 5 shows the distribution of the number of interactions per video for both the distance learners and the in-house class. The average number of interactions was 5.6 for the distance learners and 4.3 for the in-house class. A $t$-test on the two distributions gives a value of 2.3 with a critical value of 2 at $5 \%$ significance and as might be expected does give the indication that potentially there is more use by the distance learners. However, if we consider that one view is equivalent to about three interactions (Figure 2) then this is not a significant difference in the usage.

This finding links to the argument about attendance verses video use and agrees with data found in several studies arguing that students see lecture recordings as a supplement rather than a replacement to live lectures (Pearce and Scutter, 2010; Von Konsky et al., 2009); thus in this case would watch the recording of the lecture anyway. Van Zanten et al. (2012) also seemed to see little difference between the number of uses of full lecture capture and short summarised material between an in-house class and distance learners, though the distance learners, not too surprisingly, did rate both the lecture videos and short summary videos more "valuable for their understanding" most likely as a result of not having the option to attend live lectures.

\subsection{Exam Results}

As the AES2014 course had information for each individual student, we are able to examine the student exam mark against the average number of video interactions, Figure 6(a). This seems to indicate that there is a slight positive trend between the number of interactions (views) of the videos and the exam mark gained, although as expected there is a lot of scatter ${ }^{2}$. It is important to note here that there is a likelihood that higher achieving students tend to be more motivated and are thus more likely to engage with the videos more anyway. This effect has been seen by Von Konsky et al. (2009). To test this concept, the student's exam marks can be scaled by their mark

\footnotetext{
${ }^{2} R^{2}$ value is greater than critical value at a significance level of 0.01 .
} 

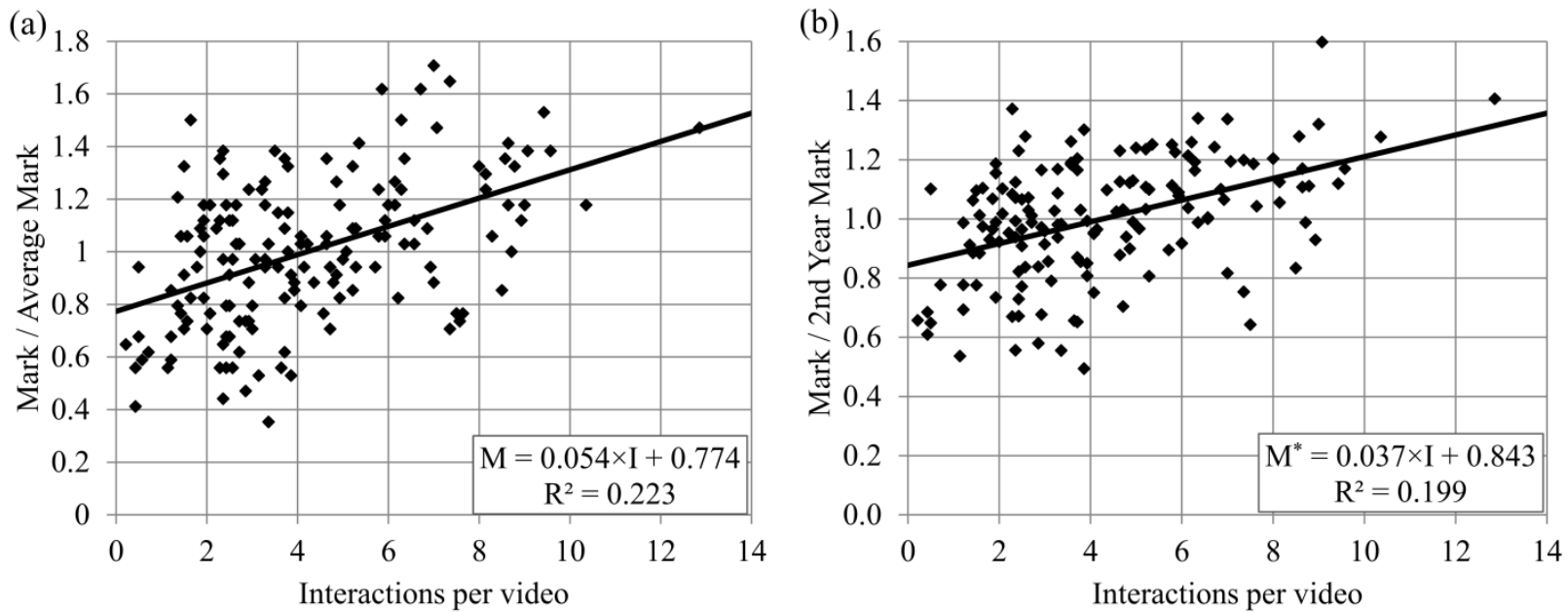

Figure 6. Interactions per video for the AES2014 lecture videos versus (a) exam mark and (b) exam mark divided by $2^{\text {nd }}$ year mark.

in second year (the previous year to this course), this will benchmark the higher achieving students, Figure 6(b). This shows that, despite the scatter, there is still an increase in marks, relative to their own performance, for the students who interact with the videos more.

Several studies have used student self-reporting in order to gain information on the effectiveness of rich-media materials; however, few have looked at exam results. Aldamen et al. (2015) state that a slight positive relationship was observed between captured lecture viewership and performance in their class. However, others state that there is essentially no difference between video users and non-users (Bosshardt and Chiang, 2016; Hadgu et al., 2016; Williams et al., 2016).

Day and Foley (2006) attempted to quantify the benefit of lecture capture material by dividing a class into two groups, a group who attended lectures as normal and a group who were offered access to lecture recordings. The group with the recordings scored over 8 marks (out of 100) higher on their final exam.

It should also be pointed out that there is no real difference between the distance learners and the in-house class in terms of average mark and mark range, Figure 7. The two cohorts are evenly distributed across the plot. 


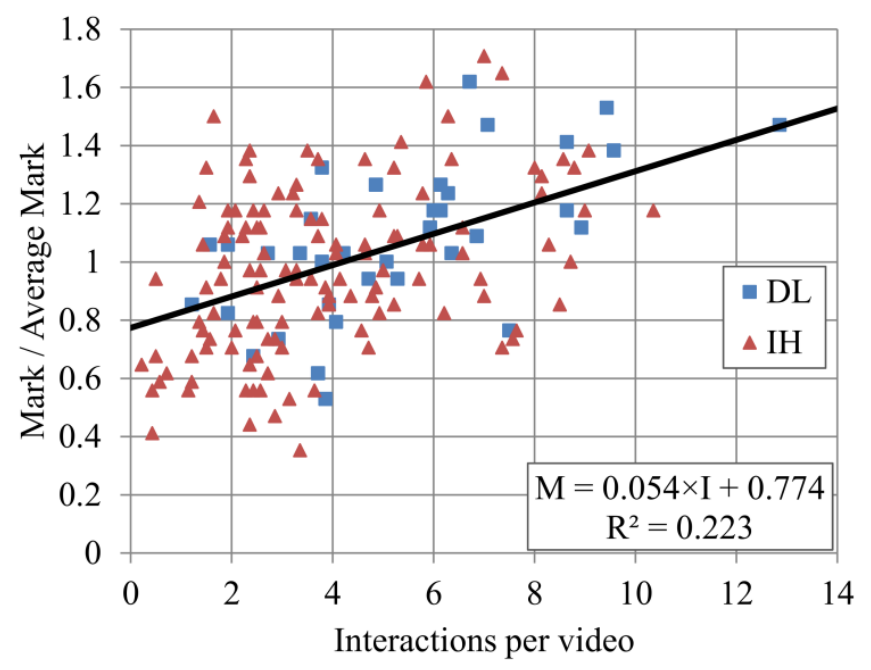

Figure 7. Exam mark vs Interactions per video for the AES2014 lecture videos with the distance learners (DL) and the in-house class (IH) displayed separately.

\subsection{Focused Activity}

If rich-media materials are to be used to their full potential they should be designed to replace some lecture material allowing time in live lectures for other activities. This means that they have to be designed with specific learning objectives in mind and potentially linked to other activities to motivate students to interact with them. LABS recently introduced some video material as a trial to some of the experiments undertaken by all the students in the class. Students are encouraged to watch the videos before they come to the laboratory, and then have the opportunity to watch the video just after the experiment, prior to their assessment of how they process their experimental data and being asked questions about the experiment they have just performed. 

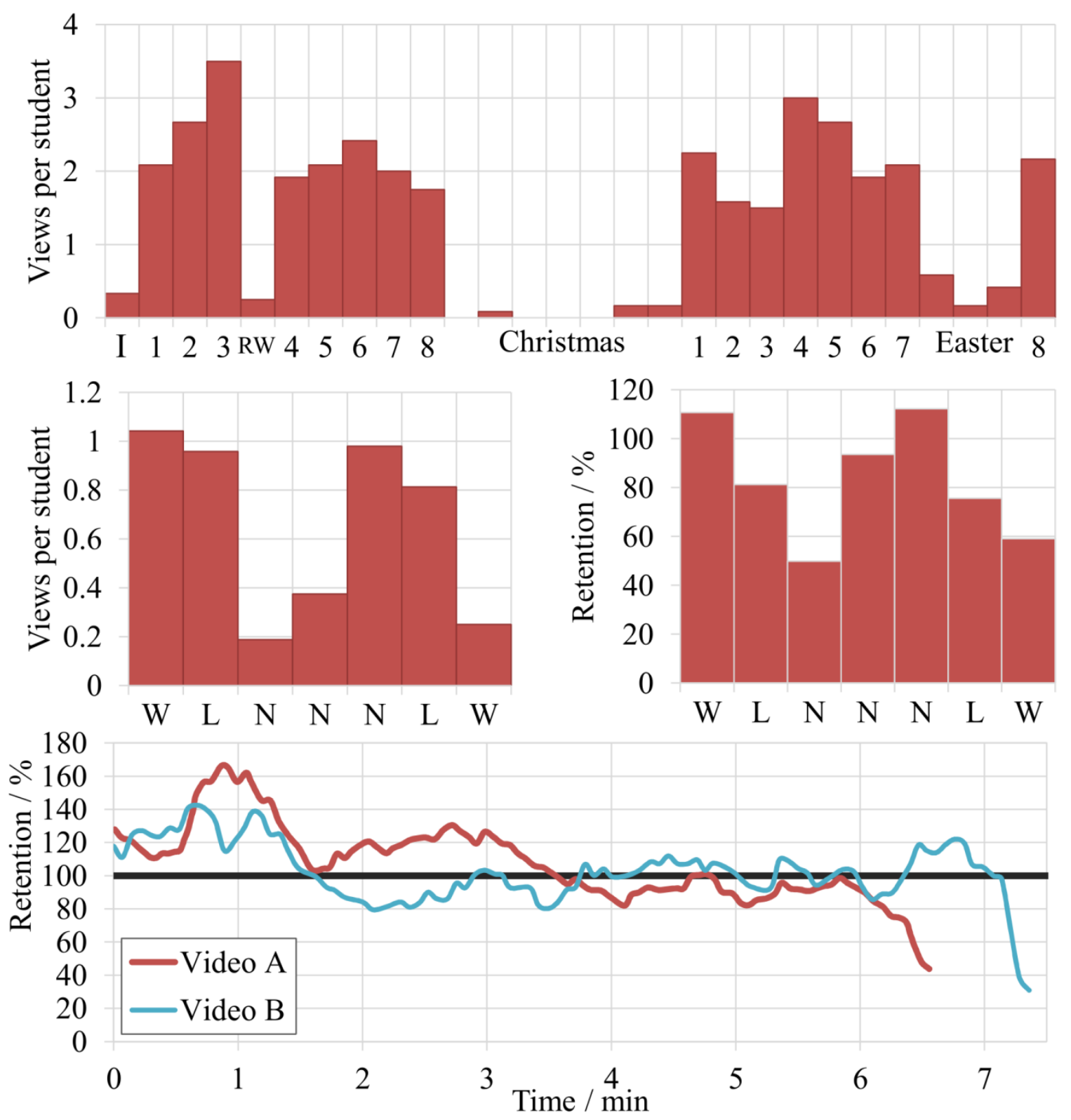

Figure 8. Weekly profile of watches averaged per video and local retention of 2 average videos. Top row - views per student per week, this data is based on the fact that 12 students are undertaking each experiment with a video per week. $I$ is induction week for the labs, and RW is reading week where there are no laboratory experiments. Centre row - views per student averaged over each day in a week, this data is based on the fact that 6 students are undertaking each experiment with a video on each laboratory day. $W$ is weekend, $L$ is lab day, and $\mathbf{N}$ is non-lab day. Bottom row - Local retention for 2 videos used for the focussed activities.

It can be seen from Figure 8 that on average each video is watched twice by the students; once before the lab, generally the day before, and once on the lab day. The retention for these videos is also very high (an average of over $90 \%$ ), with several areas of over $100 \%$ retention, where students rewind to re-watch parts of the video. 

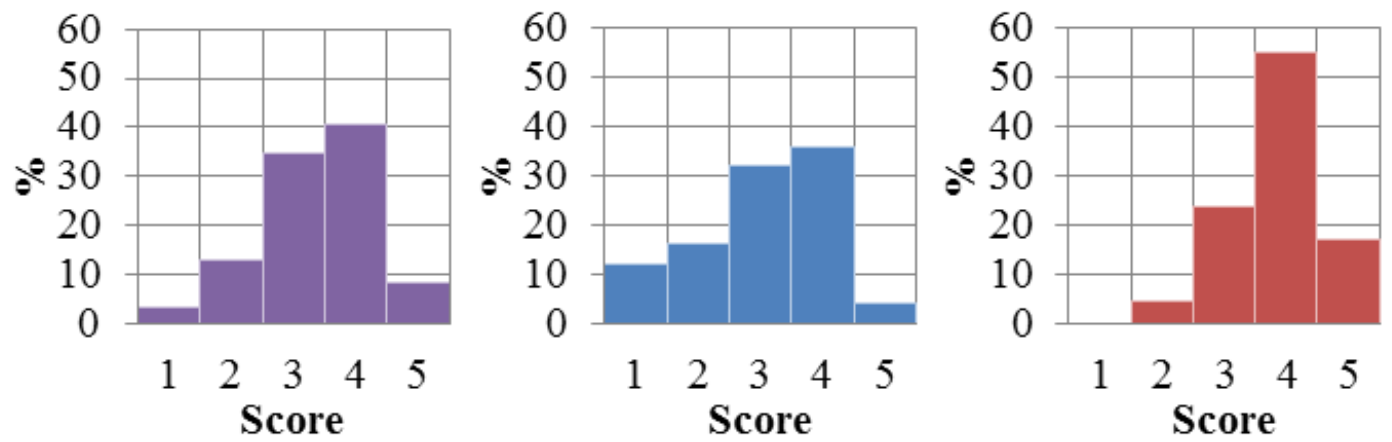

Figure 9. Survey score for "How prepared do you feel for this experiment?" where 1 is not prepared, and 5 is very prepared. Left - No video available, Centre - Did not watch video, Right - Watched video.

In the LABS class an anonymous survey was given to the students after they had finished the experiment, some of these questions were designed to assess the helpfulness of the videos and if they had used them before undertaking the experiment. Over $80 \%$ of the students in the class watched the videos before the experiment and rated the quality of the videos 4.2 out of 5 . The most important question asked to assess the video was how prepared students felt for each experiment as this was one of our key aims, Figure 9. It can be seen that there is a significant increase in preparedness for the students who have watched the videos, 3.8 out of 5, compared to 3.1 out of 5 for those that didn't watch the video ${ }^{3}$, a $p$ value of $3.5 \times 10^{-6}$ for similarity in the distributions.

The did not watch distribution is very similar to the not available distribution ${ }^{4}$, whereas the watched distribution shows a significant reduction in the amount of students who felt unprepared, in fact no student put "not prepared" after watching the videos. Three of the five LABS videos related to the assessed focused activity whereas the other two videos provided general, but necessary, information for the labs, for example on error analysis. The retention and views for the more general videos were lower than the three focused activity videos, the retention fitted in around the average line shown in Figure 4, replotted as Figure 10. Clearly the focused nature of the activity increases the views and the retention and thus could be used to replace some lecture content.

When examining student use of rich media material, it is important to think about what device students are using to access the material. YouTube provides information on the devices the videos were watched on. From Figure 11 it can be seen that over $90 \%$ of all the video views were on a computer rather than a tablet or mobile device. This is the case for both the AES course and the LABS course; there is very little difference between the two even though they are from different levels of course.

\footnotetext{
${ }^{3} 3.3$ out of 5 was the average for the labs where the videos weren't available. 3.7 out of 5 was the combined average for the Yes and No together.

${ }^{4}$ The not available distribution was also not statistically different to the distribution received for the same question in the 2014-2015 laboratory class where no videos were available for any experiment.
} 


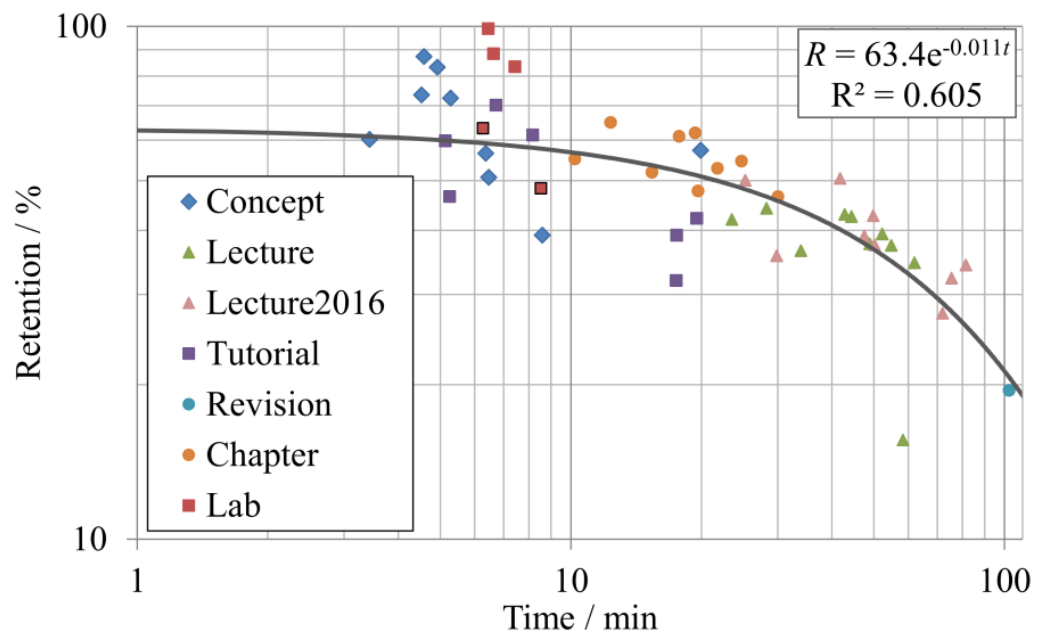

Figure 10. Retention versus video time for all videos including the laboratory videos. The laboratory videos not linked to a focused activity are outlined in black and these fall around the average line. The laboratory videos linked to a focused activity have a higher retention than expected for the length of video.
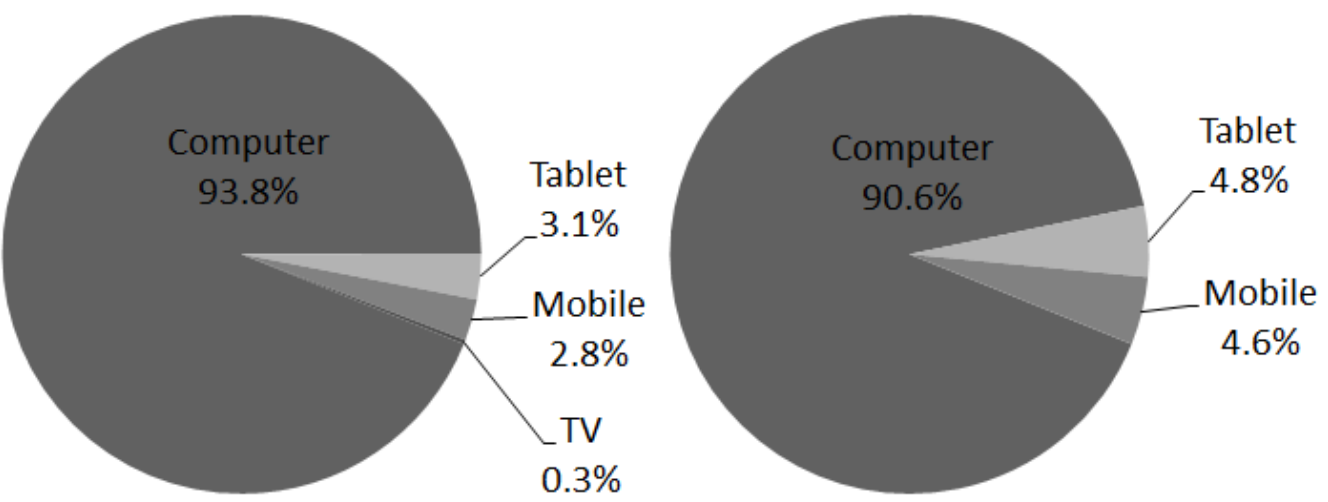

Figure 11. Devices used to view the videos, left AES and right LABS.

\section{CONCLUSIONS}

This study has presented a review of the use of five different rich-media types across a cohort of over 200 students based on two units being taken simultaneously and some focused activities for a cohort of almost 300 students. The results show that students use rich-media resources. They clearly access them for a variety of purposes, most notably to check tutorials, for coursework, and for revision. Though for these purposes, a range of rich-media resources is needed as part of a suite of learning materials. There is evidence that students use key concept videos and tutorial solution videos in a very different manner. The key-concept videos should be kept as short and focussed as possible to help deliver understanding of difficult topics, while tutorial videos will always assist those who just want to check their answers. Chapter summary videos seemed to be used by the students as longer key-concept videos with focused viewing of the part they found 
most difficult. Creating a video around a focused activity increases both the number of video views and the retention of the video.

Concerns are often given that rich-media resources reduce lecture attendance but as stated earlier the AES course attendance averaged $83 \%$ for the whole semester. However, there was little difference in the use of the rich-media resources by the students in attendance and those taking the class by distance learning. The exam mark showed a slight positive correlation with the number of video views, but it is unsure whether this is due to video use or the fact that students with higher marks tend to be more motivated so will watch videos more.

An example of the videos created and used in this paper for AES can be found at https://www.youtube.com/c/AdvancedEngineeringSeparations.

\section{REFERENCES}

Aldamen, H., Al-Esmail, R., Hollindale, J., 2015. Does Lecture Capturing Impact Student Performance and Attendance in an Introductory Accounting Course? Accounting Education 24, 291-317.

Belton, D.J., 2016. Teaching process simulation using video-enhanced and discovery/inquirybased learning: Methodology and analysis within a theoretical framework for skill acquisition. Education for Chemical Engineers 17, 54-64.

Bongey, S.B., Cizadlo, G., Kalnbach, L., 2006. Explorations in course-casting: podcasts in higher education. Campus-Wide Information Systems 23, 350-367.

Bosshardt, W., Chiang, E.P., 2016. Targeting Teaching Lecture Capture Learning: Do Students Perform Better Compared to Face-to-Face Classes? Southern Economic Journal 82, 1021-1038.

Copley, J., 2007. Audio and video podcasts of lectures for campus-based students: production and evaluation of student use. Innovations in Education and Teaching International 44, 387-399. Davis, S., Connolly, A., Linfield, E., 2009. Lecture capture:making the most of face-to-face learning. Engineering Education 4, 4-13.

Day, J., Foley, J., 2006. Evaluating web lectures: a case study from HCI, CHI '06 Extended Abstracts on Human Factors in Computing Systems. ACM, Montreal, Quebec, Canada, pp. 195200.

Fernandez, V., Simo, P., Sallan, J.M., 2009. Podcasting: A new technological tool to facilitate good practice in higher education. Computers \& Education 53, 385-392.

Franklin, D.S., Gibson, J.W., Samuel, J.C., Teeter, W.A., Clarkson, C.W., 2011. Use of Lecture Recordings in Medical Education. Medical Science Educator 21, 21-28.

Giannakos, M.N., Chorianopoulos, K., Chrisochoides, N., 2015. Making sense of video analytics: Lessons learned from clickstream interactions, attitudes, and learning outcome in a video-assisted course. The International Review of Research in Open and Distributed Learning 16.

Guertin, L.A., Bodek, M.J., Zappe, S.E., Kim, H., 2007. Questioning the student use of and desire for lecture podcasts. Journal of Online Learning and Teaching 3, 133-141.

Hadgu, R.M., Huynh, S., Gopalan, C., 2016. The Use of Lecture Capture and Student Performance in Physiology. Journal of Curriculum and Teaching 5. 
Holbrook, J., Dupont, C., 2009. Profcasts and Class Attendance - Does Year in Program Matter? Bioscience Education 13, 1-4.

Kazlauskas, A., Robinson, K., 2012. Podcasts are not for everyone. British Journal of Educational Technology 43, 321-330.

Leadbeater, W., Shuttleworth, T., Couperthwaite, J., Nightingale, K.P., 2013. Evaluating the use and impact of lecture recording in undergraduates: Evidence for distinct approaches by different groups of students. Computers \& Education 61, 185-192.

Parson, V., Reddy, P., Wood, J., Senior, C., 2009. Educating an iPod generation: undergraduate attitudes, experiences and understanding of vodcast and podcast use. Learning, Media and Technology 34, 215-228.

Pearce, K., Scutter, S., 2010. Podcasting of health sciences lectures: Benefits for students from a non-English speaking background. Australasian Journal of Educational Technology 26, 10281041.

Pursel, B., Fang, H.-N., 2012. Lecture Capture: Research and Future Directions. The Schreyer Institute for Teaching Excellence.

Sadik, A., 2015. Students' Preferences for Types of Video Lectures: Lecture Capture vs. Screencasting Recordings. International Journal of E-Learning and Distance Education 30.

Saunders, F.C., Hutt, I., 2015. Enhancing large-class teaching: a systematic comparison of richmedia materials. Higher Education Research \& Development 34, 1233-1250.

Sutton-Brady, C., Scott, K.M., Taylor, L., Carabetta, G., Clark, S., 2009. The value of using short-format podcasts to enhance learning and teaching. ALT-J 17, 219-232.

Van Zanten, R., Somogyi, S., Curro, G., 2012. Purpose and preference in educational podcasting. British Journal of Educational Technology 43, 130-138.

Von Konsky, B.R., Ivins, J., Gribble, S.J., 2009. Lecture attendance and web based lecture technologies: A comparison of student perceptions and usage patterns. Australasian Journal of Educational Technology 25, 581-595.

Williams, A.E., Aguilar-Roca, N.M., O'Dowd, D.K., 2016. Lecture capture podcasts: differential student use and performance in a large introductory course. Educational Technology Research and Development 64, 1-12. 